University of Nebraska - Lincoln

DigitalCommons@University of Nebraska - Lincoln

Educational Psychology Papers and

Publications

Educational Psychology, Department of

6-8-2003

Investigating the Acceptability of Behavioral Interventions in Applied Conjoint Behavioral Consultation: Moving From Analog Conditions to Naturalistic Settings

\author{
Richard J. Cowan \\ University of Nebraska-Lincoln, rcowan1@kent.edu \\ Susan M. Sheridan \\ University of Nebraska-Lincoln, ssheridan2@unl.edu
}

Follow this and additional works at: https://digitalcommons.unl.edu/edpsychpapers

Part of the Educational Psychology Commons

Cowan, Richard J. and Sheridan, Susan M., "Investigating the Acceptability of Behavioral Interventions in Applied Conjoint Behavioral Consultation: Moving From Analog Conditions to Naturalistic Settings" (2003). Educational Psychology Papers and Publications. 13.

https://digitalcommons.unl.edu/edpsychpapers/13

This Article is brought to you for free and open access by the Educational Psychology, Department of at DigitalCommons@University of Nebraska - Lincoln. It has been accepted for inclusion in Educational Psychology Papers and Publications by an authorized administrator of DigitalCommons@University of Nebraska - Lincoln. 


\title{
Investigating the Acceptability of Behavioral Interventions in Applied Conjoint Behavioral Consultation: Moving From Analog Conditions to Naturalistic Settings
}

\author{
Richard J. Cowan and Susan M. Sheridan \\ University of Nebraska-Lincoln
}

\begin{abstract}
Whereas there exists a vast literature investigating consumer satisfaction ratings of various behavioral interventions, the majority of these studies have been limited to analogue conditions, which may compromise utility and generalization. Additionally, most research has failed to explore multiple-source, multiple-setting data in the investigation of treatment acceptability. This study investigated parent, teacher, and child treatment acceptability ratings derived from field-based conjoint behavioral consultation cases. Data indicate that overall, parents, teachers, and children rated conjoint behavioral consultation-based behavioral interventions as very to highly acceptable. For parents, interventions with a reductive component were rated as more acceptable than interventions using both positive and negative components; no significant differences were found among teacher and child group ratings. For teachers, there was a positive relationship between (a) intervention complexity and treatment acceptability ratings and (b) problem severity ratings and treatment acceptability ratings. Additionally, regression analyses indicate that for teachers, the interaction of complexity and problem severity significantly predicted teacher treatment acceptability ratings, with teacher severity ratings demonstrating greater predictive validity. Implications of these findings and directions for future research are explored.
\end{abstract}

This article was reviewed and accepted for publication in School Psychology Quarterly under the editorship of Terry B. Gutkin.

Research described in this article was supported by federal grants awarded to Susan M. Sheridan by the U.S. Department of Education, Office of Special Education and Rehabilitative Services. The statements and opinions contained herein belong to the authors and are not reflective of the granting agency.

The authors thank several parents, teachers, and students who all contributed greatly to the completion of this study and Jenenne Geske and Kathy Shapley for their consultation regarding the statistical analyses.

Address correspondence to Richard J. Cowan, University of Nebraska-Lincoln, Department of Educational Psychology, 244 Teachers College Hall, Lincoln, NE 68588-0345; Email: psyrjc@aol.com. 
Treatment acceptability may be defined as the judgments about treatment procedures by nonprofessionals and consumers of treatment as to whether treatment is fair, reasonable, or intrusive (Kazdin, 1980). A review of the treatment acceptability literature reveals that a number of factors have been hypothesized to effect treatment acceptability and a number of models have been developed to explain the relationship of treatment acceptability to various factors within the consultation process (for a review see Eckert \& Hintze, 2000; Elliott, 1988; Reimers, Wacker, \& Koeppl, 1987).

Researchers have used analogue studies to identify factors that appear to be related to the acceptability of various interventions. Specifically, one major line of research has developed through using "type of intervention" as an independent variable (Elliott, 1988; Reimers et al., 1987). For example, several studies (Elliott, Witt, Galvin, \& Peterson, 1984; Kazdin, 1980; Witt, Elliott, \& Martens, 1984; Witt \& Robbins, 1985) have determined that positive treatments (e.g., treatments using praise, differential reinforcement, or token economies) are consistently rated by a variety of consumers (i.e., parents, teachers) as more acceptable than negative treatments (e.g., response-cost, time out, or loss of privileges).

The complexity of the intervention has also been identified as a factor potentially affecting treatment acceptability ratings (Elliott, 1988; Reimers et al., 1987), with researchers hypothesizing that in general, the more complex the intervention procedure (i.e., more steps and procedures), the less acceptable it will be perceived by treatment agents (i.e., parents and teachers) and treatment consumers (i.e., children). Additionally, researchers have concluded through analog research that in general there is a relationship between the severity of the child's behavior problem and the acceptability of the intervention. Specifically, the more severe the child's behavior problem, the more acceptable is any given intervention (Elliott et al., 1984; Witt, Moe, Gutkin, \& Andrews, 1984). In a seminal study, Elliott et al. (1984) demonstrated a relationship between these two factors: as the problem severity rate increases, so does the acceptability level of more complex interventions (i.e., interventions with multiple components), indicating an increased acceptance of (or tolerance for) more complex interventions when problem severity is deemed sufficiently high.

In a related line of research, meta-analyses by Weisz, Weiss, Alicke, and Klotz (1987) and Weisz, Weiss, Han, Granger, and Morton (1995) investigated the efficacy of interventions for children and adolescents by category (i.e., behavioral, client-centered [nondirective], insight oriented [psychodynamic], and group therapy formats). Weisz et al. (1987) reported effect size (ES) data indicating behavioral interventions to be more effective than nonbehavioral interventions ( $M$ behavioral $\mathrm{ES}=.88, M$ nonbehavioral $\mathrm{ES}=.44$ ). Although this research produced valuable information regarding the efficacy of various types of intervention (consequently prompting interventionists to use behavioral interventions), it did not consider social validity indices such as treatment acceptability. Assessing parent, teacher, and child acceptability ratings for various categories of empirically validated behavioral interventions (e.g., 
home-note, self monitoring, training/skills enhancement) may yield valuable information for practitioners working with these consumers. For example, it may help interventionists predict conditions under which interventions may not be accepted and implemented with integrity (e.g., if the intervention is too complex), allowing them to prepare proactively for such conditions.

Researchers have also attempted to provide a meaningful context through which the relationship of factors potentially impacting treatment acceptability may be better understood. For example, Witt and Elliott (1985) propose a conceptual framework that encompasses four key components: treatment acceptability, treatment integrity, treatment use, and treatment effectiveness. In their model, Witt and Elliott hypothesize that these four components are related in a dynamic, reciprocal manner in that if the individual implementing the intervention does not approve of and accept the intervention developed by the consultation team, then treatment use and treatment integrity (the degree to which treatment is delivered as intended; Yeaton \& Sechrest, 1981) may suffer. When treatment integrity is at risk, poor treatment outcomes may result. Reimers et al. (1987) add consumer treatment knowledge (i.e., the consultees' understanding of the conceptual foundations of the treatment, in addition to procedural knowledge regarding implementation) and level of disruption (i.e., the amount of disruption the intervention will likely yield) to this set of variables, potentially influencing overall treatment acceptability and impacting treatment integrity and outcome. These explanatory models may be further investigated and their relevance determined only after the nature of the relationship of various factors to treatment acceptability is investigated through context-relevant, field-based research. Clearly, additional applied research is needed to investigate the validity of hypothesized relationships between treatment acceptability, treatment integrity, and consultation outcomes.

Whereas much research exists investigating factors that potentially impact treatment acceptability (the first link in the treatment acceptability-integrity — outcome relationship theory; Witt \& Elliott, 1985), to date it has been limited to hypothetical scenarios and analog conditions (Eckert \& Hintze, 2000; Elliott, 1988; Reimers et al., 1987). Research based on hypothetical scenarios may be limited in terms of generalizability to and implications for fieldbased practice (Eckert \& Hintze, 2000; Elliott, 1988). Most research has been limited to assessing treatment acceptability from one group of consumers at a time, often relying on input from undergraduate students in university settings. Because the report of multiple relevant consumers is critical in social validity research (Kazdin, 1977, 1982), field-based research considering multiple consumers' perspectives across multiple settings would likely yield a meaningful contribution to the consultation literature (Gresham \& Noell, 1993).

In an effort to assess acceptability ratings across multiple consumers, Gray and Gutkin (2001) simultaneously surveyed teachers, parents, school administrators, and children regarding their relative acceptability ratings of a substantial list of behavioral interventions. Although this study expanded the 
literature by analyzing data from multiple raters, including school administrators-a powerful group of individuals often overlooked in consultation research-more applied research is needed to understand better the nature of multiple-source treatment acceptability ratings. Given that multiple participants (i.e., parents, teachers, and students) across multiple settings (i.e., home, school) may be involved in the consultation process, conjoint behavioral consultation (CBC; Sheridan, Kratochwill, \& Bergan, 1996) provides a service delivery model through which consumer treatment acceptability may be assessed in applied research.

\section{CBC}

CBC (Sheridan et al., 1996) is an indirect, structured model of consultation service delivery whereby parents, teachers, and support staff are joined to work together to address the academic, social, or behavioral needs of an individual for whom all parties bear some responsibility (Sheridan \& Kratochwill, 1992). Following from the behavioral consultation model (Bergan \& Kratochwill, 1990), the $\mathrm{CBC}$ problem-solving process is operationalized via four stages: (a) problem identification, (b) problem analysis, (c) treatment implementation, and (d) treatment evaluation. During problem identification, the consultation team explores shared concerns, mutually determines target behaviors for intervention, conducts a functional assessment of the targeted behavior, and determines data collection procedures across contexts (i.e., home and school). During the second stage of $\mathrm{CBC}$, problem analysis, the team reviews baseline data, conducts a data-based functional assessment of the target behavior across settings, explores hypotheses about the surrounding conditions of the behavior, and specifies procedures for a hypothesis-driven intervention plan.

Treatment implementation concerns the across-setting implementation of the mutually determined treatment plan by consultees (i.e., parents, teachers, and, in some cases, support staff). During the final stage of CBC (i.e., treatment evaluation), the consultation team evaluates the overall efficacy of the intervention. Specifically, the team determines goal attainment and assesses the desirability of retaining, modifying, or terminating the intervention, and when appropriate, planning for generalization. $\mathrm{CBC}$ is by no means static as the team progresses through the stage-wise process; rather, it is dynamic and cyclical in practice, with the flexibility of recycling through any stage to meet the needs and goals of the consultation team and child client.

The efficacy of $\mathrm{CBC}$ has received much empirical support to date (for a review of related research see Sheridan, 1997, and Sheridan et al., 1996). Specifically, research has determined $\mathrm{CBC}$ to be an effective means of service delivery when coupled with empirically validated interventions for use with elementaryaged children with social skills deficits (Colton \& Sheridan, 1998; Sheridan, Kratochwill, \& Elliott, 1990), and for middle school-aged children with academic deficits (Weiner, Sheridan, \& Jenson, 1998). Additionally, in a study re- 
porting the outcomes of a 4-year investigation of interventions applied through CBC, Sheridan, Eagle, Cowan, and Mickelson (2001) determined that behavioral outcomes across settings were highly favorable (i.e., mean $\mathrm{ES}=1.10, S D$ $=1.07, C I=.83$ to 1.36 ) for children demonstrating academic, behavioral, and social difficulties. In a line of research investigating consumer satisfaction and preference for CBC, Sheridan and Steck (1995) found that school psychologists readily endorse the problem-solving procedures of the model. Additionally, Freer and Watson (1999) found that parents and teachers rated CBC as the most acceptable form of consultation (over parent- and teacher-only consultation models). Although $\mathrm{CBC}$ research to date has demonstrated the efficacy of and preference for the model, there remains a need to explore the consumers' acceptability of behavioral interventions delivered through the $\mathrm{CBC}$ process.

\section{Purpose of Study}

A primary objective of this study was to move beyond analog conditions to investigate how multiple consumers (i.e., parents, teachers, and students) rated the acceptability of various types of behavioral interventions as implemented across naturalistic settings (i.e., home and school), within the context of CBC. Additionally, the study was designed to investigate literature-derived hypotheses regarding consumer acceptability ratings. One hypothesis was that parents, teachers, and students would rate interventions comprised of both a positive and reductive component as less acceptable than those using a positive component only (i.e., in absence of a reductive component). Another hypothesis suggested that for parents and teachers more complex interventions would be rated as less acceptable than less complex interventions. Stemming from this hypothesis was the additional prediction that for problem behaviors subjectively rated as more severe by parents and teachers, more complex interventions would be deemed more acceptable, whereas for problem behaviors subjectively rated as less severe, less complex interventions would be deemed more acceptable. It was hypothesized that as the subjective rating of the problem behavior severity increased, so would the acceptability ratings for more complex interventions (i.e., interventions with multiple components).

\section{METHOD}

This study represents a secondary research agenda stemming from a larger, multisetting, federally funded $\mathrm{CBC}$ training and research project; as such, data reported here represent a subset of a larger data set collected as part of another research project.

\section{Participants}

Participants in this study included 67 students with disabilities or those who were identified through parent or teacher report as at risk for academic 
failure due to academic, behavioral, or social deficits. The 67 students were involved in a total of $71 \mathrm{CBC}$ cases. Four of the students were included more than once in the study because more than one behavior (or academic skill) was targeted and full-scale CBC procedures were implemented for each identified target behavior. The students' parents and teachers served as consultees and 39 graduate student consultants were involved as CBC consultants. Consultation teams were comprised of a parent, classroom teacher, and consultant. Parents and teachers provided full consent to participate in this research and received a modest stipend to offset the costs of participating in the larger CBC study (e.g., child care or transportation costs). Demographic data for all participants were collected on self-report forms administered at the beginning of each consultation case. Additionally, parents reported demographic information for their children.

Child participants. Child participants included 67 students; $67 \%$ were male and $33 \%$ were female. The age range was 5 to 15 years, with a mean age of 9.7 years. Of 67 child participants, 50 were Caucasian, four were Hispanic, one was Chinese, three were African American, five were biracial, two were Native American, one was Armenian, and one was Nigerian. The child participants were attending Kindergarten through Grade 9, with a mean of Grade 4. Of these 67 students, $63 \%$ were diagnosed or classified with an academic or social-emotional disorder. Specifically, $29 \%$ were classified as learning disabled, $5 \%$ were intellectually handicapped, $44 \%$ were classified as behaviorally disordered (BD), $5 \%$ were diagnosed with attention-deficit hyperactivity disorder (ADHD), and 17\% had nonspecified or other disorders (e.g., autism, sleep disorder). A total of $37 \%$ of the students did not have a formal educational classification or psychiatric diagnosis but were considered "at risk" by the consultation team (i.e., the parent, teacher, and consultant) due to academic, behavioral, or social difficulties that were interfering with their educational progress. For these at risk students, $\mathrm{CBC}$ was provided as a prereferral intervention strategy in an attempt to prevent special education referral and classification.

Referrals to $\mathrm{CBC}$ consultants were made primarily by school psychologists, special educators, or other multidisciplinary team members. Students met the criteria for participation if school staff identified them as individuals struggling to succeed in the classroom due to (a) failure to meet basic academic requirements; or (b) the demonstration of inappropriate behaviors or social-emotional difficulties. On referral, the consultant met with the teacher and parents, explained the $\mathrm{CBC}$ model, obtained consent, and proceeded with the $\mathrm{CBC}$ process.

Parents. There were a total of 67 parents involved in the casework. A total of 45 parents $(67 \%)$ completed the Behavioral Intervention Rating Scale (BIRS; Von Brock \& Elliott, 1987). Some data were lost due to incomplete or missing data forms. A total of $76 \%$ of the parent participants were mothers and $24 \%$ of the parent participants were fathers. The average parent participant age was 37 years (range $=23$ to 54). A total of $87 \%$ of parent participants were Caucasian; $13 \%$ were from an ethnically diverse background. 
Teachers. There were a total of 67 teachers involved in CBC cases. A total of 62 teachers $(93 \%)$ completed the BIRS. Some data were lost due to incomplete or missing data forms. A total of $87 \%$ of the teacher participants were female and $13 \%$ of the teacher participants were male. The average teacher participant age was 41 years (range $=22$ to 57 ). A total of $96 \%$ of teacher participants were Caucasian; $4 \%$ were from an ethnically diverse background. The average number of years of teaching experience was 12 years $(\mathrm{range}=$ 1 to 35 ). A total of $59 \%$ of the teachers had earned a bachelor's degree; $39 \%$ had earned up to a master's degree; and $2 \%$ had earned a doctorate.

Consultants. Consultants were 39 school psychology graduate students working toward either a master's degree, an educational specialist degree (Ed.S.), or a doctorate (Ph.D.). A total of $47 \%$ of consultants had earned their bachelor's degree only; 53\% were master's level students. Of these consultants, $83 \%$ were female and $17 \%$ were male. The mean age of the consultant was 29 years (range $=23$ to 53 ). A total of $83 \%$ of consultants were Caucasian; $17 \%$ were from an ethnically diverse background.

Consultants were trained to mastery in the implementation of CBC as part of a larger, comprehensive federally funded training program. Specifically, they enrolled in a year-long advanced consultation seminar and practicum. All consultants provided consent to participate in training and research and received a stipend for their participation. Consultants were assigned school-based casework upon achieving a minimum of $85 \% \mathrm{CBC}$ interview objectives as assessed by supervisors on CBC Objectives checklists. A detailed description of the competencybased training model is beyond the scope of this article. The interested reader is referred to Kratochwill, Elliott, and Busse (1996) and Kratochwill, Sheridan, Carrington Rotto, and Salmon (1992) for demonstrations of similar models.

\section{Setting}

Consultation cases were conducted across six large school districts. Four were located in a large Western city and its suburbs and two were located in a medium-sized Midwestern city. Each of the participating schools was assigned at least one $\mathrm{CBC}$ consultant. The majority of the consultation meetings were held within the school (usually in the teacher's classroom). A negligible number of meetings were held in the home of the participant to meet the needs of individual families (e.g., lack of daycare services, cultural preference to have the meetings in their homes). Intervention procedures were implemented by parents and teachers across home and school settings.

\section{Independent Variables}

There were four independent variables considered for various analyses in this study: (a) type of behavioral intervention used for each case; (b) whether the intervention consisted of a reductive consequence (or "negative" component); (c) number of components used in the intervention; and (d) the sub- 
jective problem severity rating by the teacher and parent. Each consultation case used a specific, relevant behavioral intervention developed collaboratively and implemented by consultees across home and school settings. In addition to positive reinforcement and in some cases a reductive consequence, the types of interventions utilized included home-note, self-monitoring, and training/skills enhancement (see Table 1). All interventions involved a positive reinforcement component (i.e., verbal praise, tangible reinforcers, or a token economy system). Some interventions involved an additional reductive consequence component. Whether or not the intervention consisted of a reductive consequence served as a second independent variable. The number of components per intervention for each case was the third independent variable. Finally, parent and teacher subjective ratings of the severity of the child's target behavior served as a fourth independent variable.

\section{Dependent Variables}

Parent, teacher, and child acceptability ratings of the intervention used in $\mathrm{CBC}$ served as the dependent variables. Consultee acceptability was defined

TABLE 1. Operational Definitions of Types of Interventions

\begin{tabular}{|c|c|}
\hline Intervention Type & Operational Definition \\
\hline Home Note & $\begin{array}{l}\text { Any written means of communication between the home and school whereby } \\
\text { the student's performance as related to the target behavior is shared across } \\
\text { environments. This might include a home note specifically drawn up for the } \\
\text { purpose of sharing case-related information or sending a self-monitoring } \\
\text { (student-generated) or data collection sheet (parent-, teacher-, or consultant- } \\
\text { generated) across environments. It need not require a signature. }\end{array}$ \\
\hline
\end{tabular}

Self-Monitoring Any means by which the student monitors his or her own behavior. Selfmonitoring might include (a) having the student tally up the number of times he or she engages in a specific behavior; (b) having the student reinforce himself or herself with a sticker or a "chart move"; (c) comparison of the student's "data" with that of someone else (e.g., parent, teacher, or peer); or (d) having the student check off items on a list once he or she completes the task. This does not include when others monitor the child's behavior without the child doing so himself or herself.

Training/Skills Any means by which a student receives any form of training or practice

Enhancement related to a specific skill or target behavior. Such training might include social skills training, parent tutoring (i.e., extra help at home), modeling specific skills for the student, or using "drill and practice" procedures. This does not include merely explaining the intervention procedures to the child.

Reductive

Consequence

An event or consequence delivered by an adult after a specific undesired behavior. This consequence might be (a) the result of specific behavior(s); or (b) the result of a case in which the student does not meet a specific predetermined goal or criteria set forth by the consultation team. A reductive consequence might include time out, time away to think or problem solve, ignoring, or loss of privileges (e.g., recess, going out, television). This does not include losing previously earned "tokens," tickets, etc. 
as the subjective judgments about treatment procedures by treatment agents as to whether treatment was fair, reasonable, or intrusive (Kazdin, 1980). Child acceptability was defined as the subjective judgments about treatment procedures by consumers of treatment as to whether treatment was fair, reasonable, or intrusive (Kazdin, 1980).

\section{Instruments}

Problem severity. The problem severity rating in this study consisted of parent and teacher preconsultation ratings of the identified problem or concern. Specifically, parents and teachers were asked to rate the severity of the problem to be addressed through consultation using a 7-point Likert scale $(1=$ mild; 7 = very severe). This instrument has been used in consultation outcome research (Sheridan et al., 2001).

Treatment acceptability. Parent and teacher intervention acceptability ratings were measured via the Behavioral Intervention Rating Scale (BIRS; Von Brock \& Elliott, 1987). The BIRS consists of 24 self-report items rated on a 6-point Likert scale. Factor analysis of the BIRS yielded three factors: Acceptability, Effectiveness, and Time to Effect (Elliott \& Von Brock Treuting, 1991). In a study assessing the reliability and construct validity of the BIRS, Von Brock and Elliott (1987) reported $\alpha$ coefficients of .97 for the total scale and $.97, .92$, and .87 for the Acceptability, Effectiveness, and Time to Effect factors, respectively. The Acceptability factor, which served as the dependent measure for this research investigation, is comprised of 15 items scored on a 6-point Likert scale ( 1 = strongly disagree; 6 = strongly disagree $)$. Higher mean item scores (i.e., 5 or 6) reflect higher acceptability. Student intervention acceptability ratings were measured via the Children's Intervention Rating Profile (CIRP; Witt \& Elliott, 1985). The CIRP has been used to investigate children's perceptions of various interventions in analog conditions, with results suggesting acceptable levels of reliability and construct validity (Turco \& Elliott, 1986a, 1986b). The CIRP consists of seven self-report items rated on a 5-point Likert scale $(1=I$ agree very much, $5=I$ disagree very much), with three reverse-coded items (items 2, 3, and 4). As opposed to the BIRS, lower CIRP mean item scores (i.e., 1 or 2) reflect higher acceptability. For comparison purposes in this study, CIRP mean item scores were reversecoded so higher mean item scores (i.e., 4 or 5) reflected higher acceptability ratings. The CIRP has been used to investigate children's perceptions of various interventions in analog conditions (Turco \& Elliott, 1986a,b).

\section{Procedures}

All CBC cases were completed via the four-stage, problem-solving model (i.e., problem identification, problem analysis, treatment implementation, and treatment evaluation) described earlier. As indicated previously, data reported were collected as part of a larger $\mathrm{CBC}$ training and research project. Experi- 
mental procedures (e.g., random assignment to treatment conditions) were not used for this aspect of the study, given that the primary research project was not geared toward this specific set of hypotheses. This research project represented a secondary research agenda (i.e., to investigate how consumers rated the acceptability of interventions in naturalistic settings through the $\mathrm{CBC}$ process).

Data collection. Subjective problem severity ratings from each parent and teacher were collected at the outset of $\mathrm{CBC}$ on a preconsultation data collection form. Parent and teacher acceptability ratings were collected during the last $\mathrm{CBC}$ meeting (i.e., during treatment evaluation) on a final perceptions data collection form. Children's intervention acceptability ratings were collected by the consultant post-treatment evaluation via a single measure (i.e., the CIRP).

Coding procedures. Information regarding the specific types of interventions was gathered via case file inspection and review of case reports. Specifically, summaries of intervention procedures were retyped from case reports to simplify the intervention coding procedure. The organizational schema of this coding procedure was derived from two major sources: (a) research investigating the overall efficacy of psychological intervention (Weisz et al., 1987, 1995), and (b) evidence-based, practitioner-oriented materials for psychological intervention (Rhode, Jenson, \& Reavis, 1992; Stoner, Shinn, \& Walker, 1991). By comparing descriptions of interventions used in the $\mathrm{CBC}$ cases to the criteria used in large-scale efficacy studies (Weisz et al., 1987), the first author determined that all interventions could be categorized as primarily behavioral. Interventions were then compared to descriptions of interventions provided in practitioner-oriented materials (Stoner et al., 1991) and categorized based on the most salient feature of the intervention component (e.g., a form of self-monitoring, the use of a reductive consequence). Based on these distinctive characteristics, operational definitions used in this study (Table 1) were derived from composites of definitions contained in relevant behavioral intervention literature.

For each $\mathrm{CBC}$ case, two independent raters (i.e., educational psychology graduate students) coded the interventions into one or more of four categories (i.e., home note, self-monitoring, training/skills enhancement, and reductive consequences; see Table 1), indicated the complexity of the intervention (i.e., the number of components used in each case), and indicated whether the intervention was comprised of a reductive consequence (in addition to positive reinforcement). Coding resulted in a $\boldsymbol{\kappa}$ coefficient of .92 and a cell-by-cell interrater agreement ratio of .97 .

Data analyses. To investigate how parents, teachers, and children rated various types of behavioral interventions, descriptive statistics were calculated by intervention category (i.e., home note, self-monitoring, training/skills enhancement, reductive consequence; all interventions were comprised of a positive reinforcement component). Independent sample $t$-tests were calculated for each rater group (i.e., parents, teachers, and children) to determine whether there was a significant difference in acceptability ratings between the "positive" and "pos- 
itive with reductive component" categories of behavioral interventions. Pearson product-moment correlations were calculated to determine whether there existed a relationship between (a) intervention complexity (i.e., number of components) and treatment acceptability ratings for parents and teachers; and (b) problem severity rating and acceptability ratings for parents and teachers.

A hierarchical regression analysis was conducted to predict treatment acceptability ratings from the interaction between intervention complexity and problem severity rating (i.e., it was hypothesized that as the subjective rating of the problem behavior severity increased, so would the acceptability ratings for more complex interventions). Specifically, a two-step model with intervention complexity and severity ratings as the predictor variables and acceptability ratings as the dependent variable was constructed (i.e., the second step assessed the effect of the interaction between the predictor variables; the first step was not an interaction model). Centering was used to correct for multicollinearity by converting the independent variable scores to deviation scores (i.e., for each independent variable, the mean was subtracted from each individual score within that variable group) (Tabachnick \& Fidell, 2001). A regression model was constructed and analyzed first for parents as a group and then for teachers as a group. Because no data exist regarding each individual child's self-report of problem severity, regression analyses did not consider child participant data.

\section{RESULTS}

\section{Category of Intervention}

In general, parents, teachers, and students rated all CBC-based interventions as very to highly acceptable (see Table 2). BIRS Acceptability factor

TABLE 2. Parent and Teacher BIRS and Child CIRP Ratings by Type of Intervention

\begin{tabular}{|c|c|c|c|}
\hline Type of Intervention & Parent Ratings & Teacher Ratings & Child Ratings \\
\hline \multirow[t]{3}{*}{ Home Note } & $n=24$ & $n=30$ & $n=18$ \\
\hline & $M=5.34$ & $M=5.56$ & $M=4.10$ \\
\hline & $S D=.57$ & $S D=.51$ & $S D=.75$ \\
\hline \multirow[t]{3}{*}{ Self-Monitoring } & $n=21$ & $n=33$ & $n=19$ \\
\hline & $M=5.37$ & $M=5.44$ & $M=4.04$ \\
\hline & $S D=.53$ & $S D=.48$ & $S D=.70$ \\
\hline \multirow[t]{3}{*}{ Training } & $n=10$ & $n=14$ & $n=7$ \\
\hline & $M=5.22$ & $M=5.42$ & $M=3.86$ \\
\hline & $S D=.56$ & $S D=.41$ & $S D=.82$ \\
\hline \multirow[t]{3}{*}{ Reductive Consequence } & $n=12$ & $n=18$ & $n=8$ \\
\hline & $M=5.76$ & $M=5.53$ & $M=4.32$ \\
\hline & $S D=.31$ & $S D=.45$ & $S D=.75$ \\
\hline
\end{tabular}

Note. Parent and teacher data are based on the BIRS, with a 6-point Likert scale, with 6 being the highest acceptability rating. Child data are based on the CIRP, with a 5-point Likert scale, with 5 being the highest rating. All interventions included a positive reinforcement component. $n$-sample size in cell; $M$-mean item for the BIRS Acceptability factor or CIRP rating per category; $S D=$ Standard deviation. 
mean item scores ranged from 5.22 to 5.76 on a 6 -point Likert scale $(6=$ highest possible rating) for parents, 5.42 to 5.56 on the same scale for teachers, and CIRP mean item scores ranged from 3.86 to 4.10 on a 5-point Likert scale ( 5 = highest possible rating) for students.

\section{Type of Intervention (Positive versus Positive with Reductive Component)}

For parents as a group, there was a significant difference in acceptability ratings between the "positive" and "positive with reductive component" categories of behavioral interventions, $t(37)=-3.997, p<.05$, with the BIRS ratings favoring the latter group of interventions. There were no significant differences between these categories for teachers or children as groups. See Table 3 for the relevant descriptive statistics.

\section{Complexity of Intervention}

The average number of components per intervention was $2.46(S D=.94)$. For parents as a group, there was not a significant relationship between intervention complexity (i.e., number of components) and acceptability ratings (average parent rating $=5.37, S D=.57$ ). However, for teachers as a group, there was a significant positive relationship $(r=.278, p<.05)$ between intervention complexity and acceptability ratings (average teacher rating $=5.40, S D=.52$ ). Specifically, as intervention complexity increased, so did treatment acceptability ratings. This positive relationship was opposite the direction hypothesized.

TABLE 3. Parent and Teacher BIRS and Child CIRP Acceptability Ratings: Positive ${ }^{1}$ or Positive with Reductive Consequence ${ }^{2}$

\begin{tabular}{|c|c|c|}
\hline Source & Positive $^{1}$ & Positive with Reductive Consequence ${ }^{2}$ \\
\hline \multirow[t]{3}{*}{ Parent BIRS } & $n=33$ & $n=12$ \\
\hline & $M=5.22$ & $M=5.76$ \\
\hline & $S D=.58$ & $S D=.31$ \\
\hline \multirow[t]{3}{*}{ Teacher BIRS } & $n=44$ & $n=18$ \\
\hline & $M=5.35$ & $M=5.53$ \\
\hline & $S D=.55$ & $S D=.45$ \\
\hline \multirow[t]{3}{*}{ Child CIRP } & $n=9$ & $n=8$ \\
\hline & $M=4.48$ & $M=4.32$ \\
\hline & $S D=.69$ & $S D=.75$ \\
\hline
\end{tabular}

Note. Parent and teacher data are based on the BIRS, with a 6-point Likert scale, with 6 being the highest acceptability rating. Child data are based on the CIRP, with a 5-point Likert scale, with 5 being the highest rating. All interventions included a positive reinforcement component. $n$ - sample size in cell; $M$ - mean item for the BIRS Acceptability factor or CIRP rating per category; $S D=$ standard deviation.

'Interventions comprised of a positive reinforcement component without a reductive component.

${ }^{2}$ Interventions comprised of a positive reinforcement component in addition to a reductive component. 


\section{Problem Severity}

For parents as a group, the average severity rating was $5.12(S D=1.12$, range $=3$ to 7$)$ and there was not a significant relationship between problem severity rating and acceptability ratings (average parent rating $=5.37, S D=$ $.57)$. For teachers as a group, the average severity rating was $5.53(S D=1.02$, range $=2$ to 7$)$ and there was a significant positive relationship $(r=.338$, $p<.05$ ) between problem severity ratings and acceptability ratings (average teacher rating $=5.40, S D=.52$ ). Specifically, as problem severity ratings increased, so did treatment acceptability ratings.

\section{Relationship Between Problem Severity, Intervention Complexity, and Acceptability}

A regression analysis was used to investigate the hypothesis that as problem severity ratings increase, so would acceptability ratings for more complex interventions. Separate analyses were computed for parents and teachers as groups. Data indicated that for parents as a group, neither intervention complexity nor problem severity rating were significant predictors of treatment acceptability ratings. This is a logical outcome, given that there were no significant relationships determined between either independent variable and the dependent variable as assessed through the correlation analysis described earlier. However, for teachers as a group, an interaction model fitting intervention complexity and problem severity ratings was found to predict treatment acceptability ratings at a modest level $\left(R^{2}=.160\right.$, adjusted $\left.R^{2}=.111\right), F(3,52)=3.298, p=.027$. Additionally, data indicate that whereas teacher severity ratings are unique, significant predictors of treatment acceptability ratings, intervention complexity bears no unique contribution to the model (see Table 4). These data indicate that approximately $16 \%$ of the variance in teacher acceptability ratings may be accounted for by problem severity ratings. However, the data do not support the

TABLE 4. Summary of Hierarchical Regression Analysis for Variables Predicting Teachers' Intervention Acceptability Ratings

\begin{tabular}{lccl}
\hline Variable & $B$ & SE B & $\beta$ \\
\hline Step I & & & \\
$\quad$ Teacher Severity Rating & .16 & .06 & $.32^{*}$ \\
Intervention Complexity & .11 & .07 & .20 \\
Step 2 & & & $.30^{*}$ \\
Teacher Severity Rating & .15 & .07 & .19 \\
Intervention Complexity & .11 & .07 & .50 \\
Interaction & -.05 & .08 & \\
\hline
\end{tabular}

Note. $R^{2}=.15$ for Step $1 ; R^{2}=.007$ for Step 2 . ${ }^{*} p<.05$. 
hypothesis that as problem severity ratings increase, treatment acceptability ratings for more complex interventions also increase.

\section{DISCUSSION}

Research to date has been limited to hypothetical, analog scenarios (Eckert \& Hintze, 2000) with little emphasis on multiple source, multiple (naturalistic) setting data (Gresham \& Noell, 1993). The present study represents a unique contribution to the literature by reporting multiple-source, multiple-setting consumer treatment acceptability ratings as applied to a variety of behavioral interventions implemented through field-based conjoint behavioral consultation practice. Data from this study indicate that overall, parents, teachers, and students approve highly of a variety of specific behavioral interventions delivered through the $\mathrm{CBC}$ process, making a significant contribution to the conjoint behavioral consultation and treatment acceptability research literatures. The focus of the current study was on treatment acceptability ratings and not treatment efficacy per se and it represents part of a larger research project. However, Sheridan et al. (2001) reported CBC-based behavioral intervention mean effect sizes ranging from $1.08(S D=.82, C I=.76$ to 1.39$)$ to $1.11(S D=1.24, C I=.70$ to 1.52) across the home and school settings, respectively. Given (a) the findings of the current study and (b) the fact that Sheridan et al. (2001) reported high overall efficacy for interventions delivered across the home and school settings through $\mathrm{CBC}$, the conclusion may be made that $\mathrm{CBC}$-based interventions are both effective and deemed acceptable by parents, teachers, and children.

Whereas past analog research indicated a consumer preference for positive over negative (i.e., reductive) interventions (Elliott, et al., 1984; Kazdin, 1980; Witt, Elliott, \& Martens, 1984; Witt \& Robbins, 1985), data from the present study indicate that for parents, interventions comprised of both positive and reductive components were deemed as more acceptable than those comprised of positive interventions in absence of reductive components. It may be that in the home setting reductive consequences are more common and therefore parents have more practice with and are more comfortable using them, consequently promoting greater acceptability of such interventions. There was not a significant difference in acceptability ratings for teachers as a group. It is possible that teachers view discipline (i.e., reductive consequences) as something that should occur in the home setting; therefore, intervention comprised of both positive and reductive consequences does not stand out as a more acceptable intervention approach for this group. However, this would be a more convincing argument had teachers reported higher acceptability for positive interventions over those containing reductive consequences. Finally, there was not a significant difference in acceptability ratings for children as a group. It may be the case that children respond favorably to the opportunity to gain positive reinforcement, regardless of the use of a reductive consequence.

In this study "complexity of intervention" was operationalized as the num- 
ber of components comprising the treatment plan, following from past studies (for reviews see Eckert \& Hintze, 2000; Elliott, 1988). For parents as a group, there was not a significant relationship between intervention complexity and treatment acceptability ratings. For teachers as a group there was a significant relationship between complexity of intervention and treatment acceptability ratings. However, the pattern was the opposite of what was predicted. Instead of reflecting the pattern that as complexity increases acceptability ratings decrease, data indicated that as intervention complexity increased so did treatment acceptability ratings. One might argue that teachers are willing to implement complex interventions as needed to meet the needs of individual students. Alternatively, factors other than number of components (e.g., interventions requiring complex token economies, interventions requiring the direct instruction of specific academic or behavioral skills) may constitute complexity as perceived by treatment agents and consumers in consultation.

Data indicate that for teachers as a group, those interventions used for problem behaviors rated as more severe resulted in higher teacher treatment acceptability ratings. These findings may be indicative that teachers appreciate or tolerate otherwise unacceptable interventions for more severe problem behaviors, especially when parent and consultant support are readily available. This pattern was not evident for parents as a group. It may be the case that parents appreciate consultation to the degree that they are willing to implement interventions with integrity, regardless of their perceived rating of problem severity.

With regard to the relationship between treatment complexity, problem severity ratings, and treatment acceptability ratings, data from the current study did not support the hypothesis that as treatment severity increases, so does the acceptability of more complex interventions as supported by the Elliott et al. (1984) study. For parents as a group, there were no significant relationships between either of the predictor variables (i.e., complexity of the intervention, problem severity ratings) and treatment acceptability ratings. However, for teachers as a group, there was a relationship between both predictor variables and treatment acceptability ratings. Additionally, data indicated that for teachers as a group, as problem severity increased so did the acceptability of all interventions, with a less powerful positive relationship, albeit opposite the hypothesized direction, demonstrated between complexity and treatment acceptability ratings. Due to the inclusion of students with various levels and types of special needs within the general education classroom, it is possible that teachers are becoming more familiar with implementing relatively complex interventions. Given this pattern, especially in light of the inclusionary movement, complexity may not negatively affect treatment acceptability as once hypothesized. As previously discussed, it may be argued that teachers are willing to implement complex interventions for students who demonstrate what teachers perceive to be more pervasive, severe behavior problems, especially with the support of parents and the consultant, which communicates joint ownership of problem behaviors and solutions. 


\section{Limitations of Current Study}

One limitation of the present study is that data were not available for all participants across all variables. Specifically, for some categories of interventions (i.e., positive, positive with reductive consequences, home notes, selfmonitoring, or training/skills enhancement), all parents, teachers, and children did not complete or return treatment acceptability measures (see Tables 1, 2, and 3 for numbers of participants considered for each analysis). Although consultants make every effort to secure final perceptions data for all participants, the nature of applied research is that not all participants return all data forms. Furthermore, some parents and teachers failed to complete the problem severity rating scale at the onset of consultation services. Whereas 45 parents and 62 teachers completed treatment acceptability ratings scales, only 39 of those parents and 56 of those teachers completed problem severity ratings. This echoes the challenge of ensuring complete data sets in applied research and should remind researchers to strive toward this endeavor. Because the majority of the data were retained and because this study represents one of many for which this limitation is valid (i.e., incomplete data sets in large-scale applied research), the researchers do not perceive any indicators that response rates or patterns introduced any systematic biases to the study.

As indicated earlier, the regression analyses resulted in a significant relationship between variables for teachers as a group; no such relationship resulted for parents as a group. One limitation of the current study is that there may not have been enough power in the parent data set regression analyses to detect a relationship, should it exist. Power analysis results using the Cohen and Cohen (1983) method indicated that 44 sets of scores were necessary to achieve a power level of .80 . Although this level was achieved for teachers as a group $(N=56)$, the group for which a significant relationship was determined and reported, the minimum number of scores was not achieved for parents as a group $(N=39)$. Whereas this study represents an important benchmark in this line of research, additional studies investigating similar hypotheses with larger sample sizes would provide more context through which to interpret the results of this study as this is the very nature of regression analyses research.

An additional limitation is that there were no child problem severity rating data for analyses investigating the relationship between treatment complexity, problem severity ratings, and overall treatment acceptability ratings. This is in part due to the nature of the CBC process whereby little standardized self-report data are collected from child participants (at present, such data are limited to self-monitoring data, if they are part of the intervention, in addition to the CIRP). Additionally, because positive intervention procedures were used in all cases (including those with a reductive component), the data did not allow the investigators to explore treatment acceptability ratings dependent on the "pure" categories of positive versus negative interventions.

Another limitation of the present study is that whereas problem sever- 
ity ratings were obtained prior to intervention, treatment acceptability data were obtained postintervention only. Obtaining treatment acceptability ratings prior to and following interventions may allow researchers to better determine whether actual exposure to intervention influenced acceptability ratings. Additionally, problem severity ratings were comprised of parent and teacher responses using a one-item, Likert-type rating scale. Although this scale has been used to measure problem severity as a predictor variable in a regression analysis study with treatment outcomes as a dependent variable (Sheridan et al., 2001), some researchers argue that such scales are notoriously unreliable. However, a growing group of researchers argue that a well-defined, one-item instrument is sufficient to measure simple constructs, and multiple items may unnecessarily complicate and lengthen the measurement procedure (Braithwaite \& Scott, 1991; Gardner, et al., 1998). Further, Gardner et al. (1988) suggest that single-item scales may be valid when (a) the construct being measured is relatively uncomplicated or unidimensional; and (b) participant fatigue may negatively impact the data obtained from a larger scale. Both conditions were present in the current study. Because problem severity ratings subjectively measure a relatively simple construct and given the growing body of research in support of this approach (for a review see Gardner et $a l$. [1998]), a one-item score was used in this study. However, more research is needed to assess better the reliability of this approach to measuring subjective evaluation of problem severity ratings.

\section{Implications for Consultation Practice}

Given that (a) parents, teachers, and children alike rate all categories of behavioral interventions (i.e., home notes, self-monitoring, training, and reductive consequences) as very to highly acceptable; and (b) recent research has determined the overall efficacy (Sheridan et al., 2001) and superiority (Weisz et al., 1987, 1995) of behavioral interventions, applying behavioral interventions within the conjoint behavioral consultation model will likely result in both acceptable and efficacious treatment outcomes. Additionally, given that for parents as a group interventions comprised of both positive reinforcement and a reductive consequence (i.e., time out, time away to think or problem solve, ignoring, or loss of privileges) were rated as more acceptable than those not containing a reductive consequence, consultants may wish to support the use of a reductive component in the home setting as part of the home-based intervention. However, consultants should bear in mind that these results indicate a preference for interventions comprised of both positive and negative consequences, and that practice from research would prescribe that positive consequences be used as part of the intervention. Another implication for consultation practice comes from the finding that all consumers rated the "home note" category as a highly acceptable intervention. Related to the goals of (a) joint ownership for problem identification and solution; and (b) increased communication across 
settings, as outlined in the CBC manual (Sheridan et al., 1996), using home notes appears to be a nonintrusive, highly acceptable practice that may increase treatment efficacy and improve communication across settings.

Nearly $16 \%$ of variability in teacher intervention acceptability ratings can be accounted for by the level of problem severity rating, with teachers finding interventions to be most acceptable for severe target behaviors. Teachers who are part of the $\mathrm{CBC}$ process may find interventions to be most acceptable for severe behaviors because of more resources available for intervention (e.g., consultant support in preparing for intervention, parent support in promoting a consistent intervention in the home setting). If this is the case, parents and consultants should offer to assist teachers by providing time and material resource support. Given that teachers report higher acceptability ratings for interventions focusing on more severe problem behaviors and considering the Freer and Watson (1999) findings that parents and teachers prefer CBC over other forms of consultation, joining parents and teachers as a team to problem solve across settings appears to be in the best interest of all parties.

\section{Directions for Future Research}

As with many scientific inquiries, this exploratory study yields as many questions as it does answers. Specifically, what other variables might be impacting treatment acceptability ratings, resulting in such elevated ratings? Is there something about the $\mathrm{CBC}$ process that promotes higher overall acceptability ratings? Is there something about the operationalization of the constructs (i.e., complexity) that limits variability in ratings? Another question to consider is how this study is different from analogue studies. For example, in analog studies there is control derived from random assignment into independent variable groups (i.e., positive versus negative interventions, various levels of intervention complexity). If this study were replicated using such experimental control, with equal cell sizes for each category, and with matched child participant characteristics in each group, would the outcome be more congruent with past research (e.g., consumers prefer positive over reductive procedures)? Would more variance emerge across groups of raters? Or, would differences between analog and naturalistic studies replicate? One might speculate that the current findings elucidate patterns reflective of actual preferences. Given that this is the first study to identify consumer acceptability patterns in naturalistic settings, this hypothesis appears to warrant investigation through replication studies. If such issues could be addressed, future studies may result in greater variability in treatment acceptability ratings across independent variables, thus allowing for prediction models to be constructed in relation to treatment acceptability.

It may be beneficial for future related studies to include additional categories of independent variables in the research design. For example, is it possible that the nature of the target behavior (i.e., academic skill deficit, problem 
behavior, or social-emotional difficulties) affects consumer treatment acceptability ratings? Additionally, is it possible that gender, age, or disability category impacts the treatment acceptability ratings of various consumers? In a study assessing the overall efficacy of CBC, Sheridan et al. (2001) investigated a prediction (regression) model for case outcomes (i.e., effect sizes) based on the following independent variables: client age, case complexity, and severity of problems. Results indicated that client age and problem severity predicted effect sizes in the school setting relatively well, suggesting higher effect sizes for younger individuals with more severe problems and for older individuals with less severe problems. Research investigating the relationship between these alternative independent variables and consumer acceptability ratings may yield useful results.

Clearly more research is needed to reach sound conclusions regarding the relationship between independent variables (e.g., type of intervention, complexity of intervention) and consumer treatment acceptability ratings. Such information will be necessary if prediction models are to be attained. Furthermore, such information is prerequisite to assessing the validity of this first link in the treatment acceptability-treatment integrity-treatment outcome model as proposed by Witt and Elliott (1985). Only then can researchers begin to build factors such as treatment integrity considerations into this everexpanding prediction model. From a scientist-practitioner standpoint, the ideal would be not to create a cookbook approach to consultation and behavioral intervention; rather, the goal would be to provide scientist-practitioners with as much information as possible to ascertain the greatest level of treatment integrity and outcome, ultimately benefiting students and other consumers of consultative intervention.

\section{REFERENCES}

Bergan, J. R., \& Kratochwill, T. R. (1990). Behavioral consultation and therapy. New York: Plenum.

Braithwaite, V. A., \& Scott, W. (1991). Values. In J. P. Robinson \& P. R. Shaver (Eds.), Measures of personality and social psychological attitudes (pp. 83-97). San Diego, CA: Academic Press.

Cohen, J., \& Cohen, P. (1983). Applied multiple regression/correlation analysis for the behavioral sciences (2nd ed.). Mahwah, NJ: Erlbaum.

Colton, D., \& Sheridan, S. M. (1998). Conjoint behavioral consultation and social skills training: Enhancing the play behavior of boys with attention-deficit hyperactivity disorder. Journal of Educational and Psychological Consultation, 9, 3-28.

Eckert, T. L., \& Hintze, J. M. (2000). Behavioral conceptualizations and applications of treatment acceptability: Issues related to service delivery and research methodology. School Psychology Quarterly, 15, 123-148.

Elliott, S. N. (1988). Acceptability of behavioral treatments: Review of variables that influence treatment selection. Professional Psychology: Research and Practice, 19, 68-80.

Elliott, S. N., \& Von Brock Treuting, M. (1991). The behavior intervention rating scale: Devel- 
opment and validation of a pretreatment acceptability and effectiveness measure. Journal of School Psychology, 29, 43-51.

Elliott, S. N., Witt, J. C., Galvin, G., \& Peterson, R. (1984). Acceptability of positive and reductive interventions: Factors that influence teachers' decisions. Journal of School Psychology, 22, 353-360.

Freer, P., \& Watson, T. S. (1999). A comparison of parent and teacher acceptability ratings of behavioral and conjoint behavioral consultation. School Psychology Review, 28, 672-684.

Gardner, D. G., Cummings, L. L., Dunham, R. B., \& Pierce, J. L. (1998). Single-item versus multiple-item measurement scales: An empirical comparison. Educational \& Psychological Measurement, 58, 898-912.

Gray, C. L., Gutkin, T. B., \& Riley, T. (2001). Acceptability of reward among high school teachers, parents, students, and administrators: Ecological implications for consultation at the high school level. Journal of Educational and Psychological Consultation, 12, 25-43.

Gresham, F. M., \& Noell, G. H. (1993). Documenting the effectiveness of consultation outcomes. In J. E. Zins, T. R. Kratochwill, \& S. N. Elliott (Eds.), Handbook of consultation services for children (pp. 249-273). San Francisco: Jossey-Bass.

Kazdin, A. E. (1977). Assessing the clinical or applied significance of behavioral change through social validation. Behavior Modification, 1, 427-452.

Kazdin, A. E. (1980). Acceptability of alternative treatments for deviant child behavior. Journal of Applied Behavior Analysis, 13, 259-273.

Kazdin, A. E. (1982). Single-case research designs. New York: Oxford University Press.

Kratochwill, T. R., Elliott, S. N., \& Busse, R. T. (1996). Behavioral consultation: A five-year evaluation of consultant and client outcomes. School Psychology Quarterly, 10, 87-117.

Kratochwill, T. R., Sheridan, S. M., Carrington Rotto, P. J., \& Salmon, D. (1992). Preparation of school psychologists to serve as consultants for teachers of emotionally disturbed children. School Psychology Review, 20, 530-550.

Reimers, T. M., Wacker, D. P., \& Koeppl, G. (1987). Acceptability of behavioral interventions: A review of the literature. School Psychology Review, 16, 212-227.

Rhode, G., Jenson, W. R., \& Reavis, H. K. (1992). The tough kid book: Practical classroom management strategies. Longmont, $\mathrm{CO}$ : Sopris West.

Sheridan, S. M. (1997). Conceptual and empirical bases of conjoint behavioral consultation. School Psychology Quarterly, 12, 119-133.

Sheridan, S. M., \& Colton, D. L. (1994). Conjoint behavioral consultation: A review and case study. Journal of Educational and Psychological Consultation, 5, 211-228.

Sheridan, S. M., \& Kratochwill, T. R. (1992). Behavioral parent-teacher consultation: Conceptual and research considerations. Journal of School Psychology, 30, 117-139.

Sheridan, S. M., \& Steck, M. C. (1995). Acceptability of conjoint behavioral consultation: A national survey of school psychologists. School Psychology Review, 24, 633-647.

Sheridan, S. M., Kratochwill, T. R., \& Bergan, J. R. (1996). Conjoint behavioral consultation: A procedural manual. New York: Plenum.

Sheridan, S. M., Kratochwill, T. R., \& Elliott, S. N. (1990). Behavioral consultation with parents and teachers: Delivering treatment for socially withdrawn children at home and school. School Psychology Review, 19, 33-52.

Sheridan, S. M., Eagle, J. W., Cowan, R. J., \& Mickelson, W. (2001). The effects of conjoint behavioral consultation: Results of a 4-year investigation. Journal of School Psychology, 39, $361-385$. 
Stoner, G., Shinn, M. R., \& Walker, H. M. (Eds.). (1991). Interventions for achievement and behavior problems. Silver Springs, MD: National Association of School Psychologists.

Tabachnick, B. G., \& Fidell, L. S. (2001). Using multivariate statistics (4th ed). Boston: Allyn \& Bacon.

Turco, T. L., \& Elliott, S. N. (1986a). Assessment of student acceptability ratings of teacher-initiated interventions for classroom misbehavior. Journal of School Psychology, 24, 277-283.

Turco, T. L., \& Elliott, S. N. (1986b). Students' acceptability ratings of interventions for classroom misbehaviors: A study of well-behaving and misbehaving youth. Journal of Psychoeducational Assessment, 4, 281-289.

Von Brock, M., \& Elliott, S. N. (1987). Influence and treatment effectiveness information on the acceptability of classroom interventions. Journal of School Psychology, 25, 131-144.

Weiner, R. K., Sheridan, S. M., \& Jenson, W. R. (1998). The effects of conjoint behavioral consultation and a structured homework program on math completion and accuracy in junior high school students. School Psychology Quarterly, 13, 281-309.

Weisz, J. R., Weiss, B., Alicke, M. D., \& Klotz, M. L. (1987). Effectiveness of psychotherapy with children and adolescents: A meta-analysis for clinicians. Journal of Consulting and Clinical Psychology, 55, 542-549.

Weisz, J. R., Weiss, B., Han, S., Granger, D. A., \& Morton, T. (1995). Effects of psychotherapy with children and adolescents revisited: A meta-analysis of treatment outcome studies. Psychological Bulletin, 117, 450-468.

Witt, J. C., \& Elliott, S. N. (1985). Acceptability of classroom intervention strategies. In T. R. Kratochwill (Ed), Advances in school psychology (Vol. 4, pp. 251-288). Mahwah, NJ: Erlbaum.

Witt, J. C., \& Robbins, J. R. (1985). Acceptability of reductive interventions for the control of inappropriate child behavior. Journal of Abnormal Child Psychology, 13, 59-67.

Witt, J. C., Elliott, S. N., \& Martens, B. K. (1984). Factors affecting teachers' judgments of the acceptability of behavioral interventions: Time involvement, behavior problem severity, type of intervention. Behavior Therapy, 15, 204-209.

Witt, J. C., Moe, G., Gutkin, T. B., \& Andrews, L. (1984). The effect of saying the same thing in different ways: The problem of language and jargon in school-based consultation. Journal of School Psychology, 22, 361-367.

Yeaton, W. H., \& Sechrest, L. (1981). Critical dimensions in the choice and maintenance of successful treatment: Strength, integrity, and effectiveness. Journal of Consulting and Clinical Psychology, 49, 156-167. 